\title{
Design and Simulation of Finite-time Convergence with Impact angle constraint Sliding-mode Guidance Law
}

\author{
Kai Zhang, Suo-chang Yang, Kuan-qiao Zhang, Yun-zhi Yao, Peng Chen \\ Department of Missile Engineering, Ordnance Engineering College, Shijiazhuang \\ 050003, China \\ zhangkai_blue@126.com
}

Keywords: Impact angle constraint; Finite-time convergence; Sliding-mode guidance law;

\begin{abstract}
For missile falling angle constraint targets to prevent target evasive maneuvers, missile target relative motion relationship is established, based on the sliding mode variable structure theory, the linear sliding surface, select adaptive power reaching law is proposed with the drop angle constraint of sliding mode guidance law. By using Lyapunov theory guidance law of stability analysis, the analysis of the guidance law with finite time convergence. Simulation results show that the designed guidance law is compared to the traditional guidance law, the performance is more superior, has strong robustness, meet the design requirements.
\end{abstract}

\section{Introduction}

In modern war, as the missile weapons, with precise target effect, if at a certain angle to hit the target, will realize the killing effect of warhead more. Since the scholars Kim and Grider for the first time in missile reentry guidance of angular constraint problems, more and more scholars and experts in different background, according to the the design of a variety of different theoretical methods guidance law with impact angle constraint [1-3]. Above guidance law put forward in the design of the angular constraint requirements, simulation studies have shown it to meet the requirements in terms of performance, but the guidance system for the issue of convergence over time literature really did not mention. Thus, the angular constraint guidance law with finite period of time will be combined with the new direction of research [4].

\section{The theoretical knowledge}

Definition1

Consider the following nonlinear system [4]:

$\dot{x}=f(x, t), f(0, t)=0, x \in R^{n}$

Among them, $f: U_{0} \times R \rightarrow R^{n}$ in $U_{0} \times R$ is continuous, and $U_{0}$ is the origin of $x=0$ an open neighborhood.

In the definition of a nonlinear system (1) the equilibrium point $X=0$ (local) finite time convergence, is that of arbitrary initial time $t_{0}$ and the given initial state $x\left(t_{0}\right)=x_{0} \in U$ and in the presence of a dependency $x_{0}$ on the stopped time $T \geq 0$, makes the system of equations (1) $x_{0}$ to the initial state solution $x(t)=\varphi\left(t ; t_{0}, x_{0}\right)$ is defined (and possibly not only), and

$$
\left\{\begin{array}{c}
\lim _{t \rightarrow T\left(x_{0}\right)} \varphi\left(t ; t_{0}, x_{0}\right)=0 \\
\text { if } t>T\left(x_{0}\right) \text {, than } \varphi\left(t ; t_{0}, x_{0}\right)=0
\end{array}\right.
$$

When $t \in\left[t_{0}, T\left(x_{0}\right)\right], \varphi\left(t ; t_{0}, x_{0}\right) \in U /\{0\}$ in addition, the equilibrium point $x=0$ (local) finite time stability of this system is that it is Lyapunov stable and finite time convergence in a 
neighborhood $U \in U_{0}$ of the origin. If the origin $U \in R^{n}$ is the equilibrium point of the global finite time stability.

Based on the finite time control theory, There are the following lemma:

Lemma 1 consider the nonlinear system (1), assume the existence of a definition in the neighborhood $\hat{U} \subset U_{0} \subset R^{n}$ of the origin of the smooth function $V(x)$, and there are real $c>0$ and $0<\lambda<1$ the $V(x)$ in $\hat{U}$ is positive and $\dot{V}(x)+c V^{\lambda}(x) \operatorname{in} \hat{U}$ is negative, the origin of the system is finite time stable. Stable time is determined by the initial value $x(0)=x_{0}$ and the upper bound for

$T_{x}\left(x_{0}\right)=\frac{V^{1-\lambda}\left(x_{0}\right)}{c(1-\lambda)}$

Proof: $V(x)$ in $\hat{U}$ is positive definite and $\dot{V}(x)+c V^{\lambda}(x)$ in $\hat{U}$ is upper semi negative definite

$$
\dot{V}(x) \leq-c V^{\lambda}(x), \forall t \geq 0
$$

On the integral solution can be got

$$
\left\{\begin{array}{l}
V^{1-\lambda}(x) \leq V^{1-\lambda}\left(x_{0}\right)-c(1-\lambda) t, \\
0 \leq t \leq T_{x}\left(x_{0}\right)
\end{array}\right.
$$

when $t \geq T_{x}\left(x_{0}\right), V(x)=0$, The finite convergence time of the system can be obtained

$$
T_{x}\left(x_{0}\right) \leq \frac{V^{1-\lambda}\left(x_{0}\right)}{c(1-\lambda)}
$$

The proof is complete.

\section{Relative motion model of missile and target}

Before the design of the guidance law, the need to establish a two-dimensional model of the relative motion of the missile and the target. For the convenience of analysis, the assumption that the missile and the target are particles, and the velocity of the missile is greater than the target velocity, as a constant.

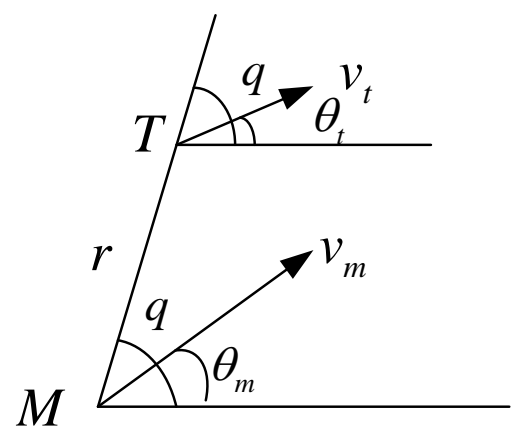

Fig. 1 Schematic diagram of the relative motion of projectile

Among them, missile $M$ and $T$ are considered as a particle; $v_{m}$ is the missile speed, $v_{t}$ as speed of the target; $\theta_{m}$ as missile trajectory angle, $\theta_{t}$ as the target heading angle; $r$ between the missile target relative distance, $q$ as angle of missile target line of sight (LOS), the provisions of all angles is a counterclockwise direction, otherwise it is negative.

Relative motion equation of projectile: 


$$
\begin{aligned}
& \dot{r}=-v_{m} \cos \left(q-\theta_{m}\right)+v_{t} \cos \left(q-\theta_{t}\right) \\
& r \dot{q}=v_{m} \sin \left(q-\theta_{m}\right)-v_{t} \sin \left(q-\theta_{t}\right) \\
& \dot{\theta_{m}}=a_{m} / v_{m} \\
& \dot{\theta}_{t}=a_{t} / v_{t}
\end{aligned}
$$

Among them, $a_{m}$ and $a_{t}$ as the missile and the target of the normal acceleration is expressed, respectively.

The formula derivation, available:

$$
\begin{aligned}
& \ddot{r}=-\dot{q}\left[v_{t} \sin \left(q-\theta_{t}\right)-v_{m} \sin \left(q-\theta_{m}\right)\right]+\dot{v}_{t} \cos \left(q-\theta_{t}\right) \\
& +v_{t} \dot{\theta}_{t} \sin \left(q-\theta_{t}\right)-\dot{v}_{m} \cos \left(q-\theta_{m}\right)-v_{m} \dot{\theta}_{m} \sin \left(q-\theta_{m}\right) \\
& \dot{r} \dot{q}+r \ddot{q}=\dot{q}\left[v_{m} \cos \left(q-\theta_{m}\right)-v_{t} \cos \left(q-\theta_{t}\right)\right]+\dot{v}_{m} \sin \left(q-\theta_{m}\right) \\
& -v_{m} \cos \left(q-\theta_{m}\right) \dot{\theta}_{m}-\dot{v}_{t} \sin \left(q-\theta_{t}\right)+v_{t} \cos \left(q-\theta_{t}\right) \dot{\theta}_{t}
\end{aligned}
$$

Put The type of substitution (6) in (11) available $\dot{r} \dot{q}+r \ddot{q}=-u_{q}+w_{q}$

$u_{q}$ and ${ }^{w_{q}}$ respectively for the missile and the target acceleration in the line of sight component on the normal vector.

Combining with (12)

$\ddot{q}=-\frac{2 \dot{r} \dot{q}}{r}+\frac{w_{q}}{r}-\frac{u_{q}}{r}$

In the terminal guidance,

$$
\begin{aligned}
& \dot{r(t)}<0, \quad 0 \leq r(t) \leq r, \quad \forall t>0 \\
& \text { select } x=\dot{q}, \quad u=u_{q} w=w_{q} \\
& \dot{x}=-\frac{2 \dot{r}}{r} x+\frac{w}{r}-\frac{u}{r}
\end{aligned}
$$

\section{Guidance law design analysis}

\section{Guidance law design}

In order to accurately hit the target, and obtain the desired Angle, according to the guidance weapon terminal guidance problem, can know the line of sight angular rate to zero on behalf of the missile hit the target under ideal condition, the constraint conditions $\left|q(t)-q_{d}\right|=0$ to be fulfilled are given in the end, so take sliding mode switching function for

$$
s=x+\frac{\lambda v_{m}}{r}\left(q(t)-q_{d}\right)
$$

Among them, $\lambda>0$ and $q_{d}$ as constraint Angle. 
Based on sliding mode variable structure control theory, in order to make the system state speeding and reaching the sliding along the sliding mode surface, selecting the adaptive exponential reaching law for $\dot{S}=-\frac{\varepsilon}{r}|s|^{\alpha} \operatorname{sgn}(s)$, type: $\varepsilon>0,0<\alpha<1$.

By the selection of reaching law

$$
\dot{s}=-\frac{\varepsilon}{r}|s|^{\alpha} \operatorname{sgn}(s) \cdot s
$$

Due to the

$$
\operatorname{sgn}(s) \cdot s=\left\{\begin{array}{l}
s, s>0 \\
-s, s<0
\end{array}\right.
$$

Namely

$$
\operatorname{sgn}(s) \cdot s=|s|
$$

So there are

$$
s \dot{s}=-\frac{\varepsilon}{r}|s|^{\alpha+1}
$$

Considering the guidance phase $\alpha>0$

Therefore,

$$
s \dot{S}=-\frac{\varepsilon}{r}|s|^{\alpha+1}<0
$$

Therefore, reaching slide condition system can reach the sliding surface.

For selection of the sliding mode surface, such as type (15), the derivative

$$
\dot{s}=\left(\dot{x}+\frac{\lambda v_{m} \dot{q}}{r}-\frac{\lambda v_{m} \dot{r}\left(q(t)-q_{d}\right)}{r^{2}}\right)
$$

Will type (16) into type (18), available

$$
\dot{x}+\frac{\lambda v_{m} \dot{q}}{r}-\frac{\lambda v_{m} \dot{r}\left(q(t)-q_{d}\right)}{r^{2}}=-\frac{\varepsilon}{r}|s|^{\alpha} \operatorname{sgn}(s) \cdot s
$$

By the type available

$$
0=-\frac{2 \dot{r}}{r} x+\frac{w}{r}-\frac{u}{r}+\frac{\varepsilon}{r}|s|^{\alpha} \operatorname{sgn}(s) \cdot s+\frac{\lambda v_{m} \dot{q}}{r}-\frac{\lambda v_{m} \dot{r}\left(q(t)-q_{d}\right)}{r^{2}}
$$

To sort out the

$$
u=-2 \dot{r} x+w+\varepsilon|s|^{\alpha} \operatorname{sgn}(s)+\lambda v_{m} \dot{q}-\frac{\lambda v_{m} \dot{r}\left(q(t)-q_{d}\right)}{r}
$$

The guidance law expression shows that the guidance law for variable structure control and the parameter perturbation and external disturbance robust $\operatorname{sgn}(s)$, switching function is contained in the variable structure guidance law, the need to constantly switch control quantity, and in the actual system, there is a certain amount of time lag, can cause a shaking of the actuator, a shaking of the missile is the jitter is actually, if the wobble is too big, can affect the accuracy of its hit target. Variable structure guidance law and jitter is the inherent shortcomings, can't eliminate, only to decrease it. So in this guidance law, symbolic function $\frac{s}{s+\delta}$ is replaced by a saturation function $\operatorname{sgn}(s)$ with high gain, the effect of reducing jitter [5]. Such as type (22)

$$
u=-2 \dot{r} x+w+\varepsilon|s|^{\alpha} \frac{s}{|s|+\delta}+\lambda v_{m} \dot{q}-\frac{\lambda v_{m} \dot{r}\left(q(t)-q_{d}\right)}{r}
$$


For play yard distance $r(t)$ and distance rate $\dot{r}(t)$, active homing missile can be measured or calculated by, while the passive homing missile can not directly get, need for real-time estimation. If the target is maneuvering target, then its acceleration $w$ generally cannot get accurately, therefore, it can be seen as interference estimate the upper bound. Mobility poor target for sea and on the ground, can be directly hypothesis $w=0$, this guidance law was

$u=-2 \dot{r} x+\varepsilon|s|^{\alpha} \frac{s}{|s|+\delta}+\lambda v_{m} \dot{q}-\frac{\lambda v_{m} \dot{r}\left(q(t)-q_{d}\right)}{r}$

\section{Finite time convergence analysis}

System motion state can be divided into phases and reaching slide along the sliding mode motion stage, because the selection of guidance law of sliding mode surface for linear sliding mode surface, so you just need to arrive in stage of sliding mode for finite time convergence analysis.

For reaching slide stage, the Lyapunov function $V_{1}=s^{2}$ is selected, its derivation, too

$$
\dot{V}_{1}=2 s \dot{s}=2 s \dot{s}=-2 k_{1}|s|^{\alpha+1}-2 k_{2} s^{2}<0
$$

In the terminal guidance,

$\dot{r(t)}<0, \quad 0 \leq r(t) \leq r, \quad \forall t>0$

select

$\dot{V}_{1}=-2 k_{1} V_{1}^{\frac{1}{2}(\alpha+1)}-2 k_{2} V_{1} \leq-2 k_{1} V_{1}^{\frac{1}{2}(\alpha+1)}, \forall t>0$

By the lemma 1 , system status, converge to the sliding surface in finite time for time

$T_{1} \leq \frac{V_{1}^{\frac{1}{2}(\alpha+1)}\left(x_{0}, 0\right)}{2 k_{1}(1-\alpha)}$

In conclusion shows that the designed guidance law can make guidance system state of convergence in a limited time.

\section{The results of simulation}

In this paper, design of finite time convergence (FTCG) guidance law with fall Angle constraint is mainly used in fixed target, to verify the precision guidance performance, using MATLAB simulink modeling capabilities to validate the designed guidance law, and with the improvement of the traditional proportional guidance law biased proportional guidance law (BPNG) were compared. Selection of the initial position of missile $(\mathrm{Xm} 0 \mathrm{Ym} 0)=(0 \mathrm{~m}, 0 \mathrm{~m})$, target initial position for (Xt0 $\mathrm{Yt} 0)=(0 \mathrm{~m}, 2000 \mathrm{~m})$, the speed of the missile is $200 \mathrm{~m} / \mathrm{s}$, incident Angle of constraint of $50^{\circ}$, the simulation results are shown in figure 2-5.

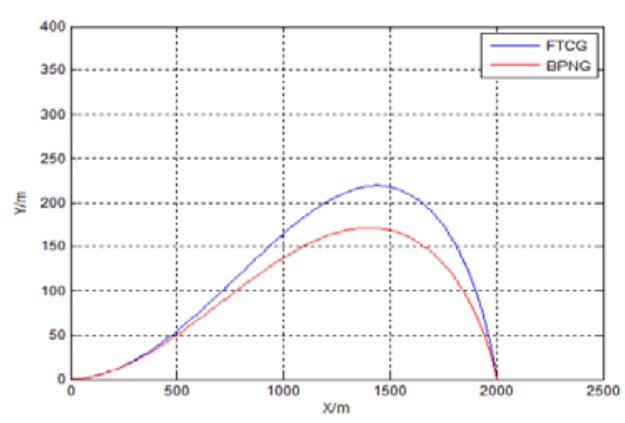

Fig.2 Missile trajectory curve

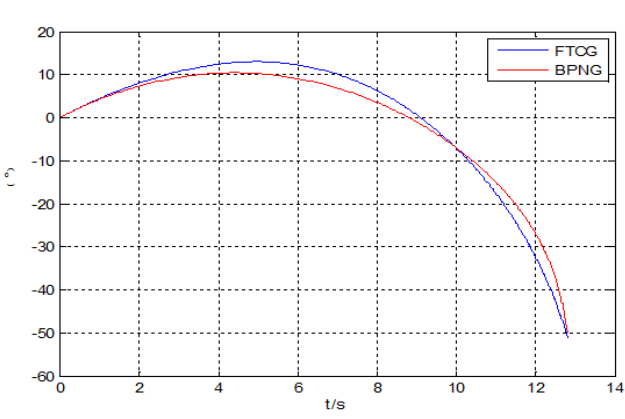

Fig. 3 Elevation Angle diagram 


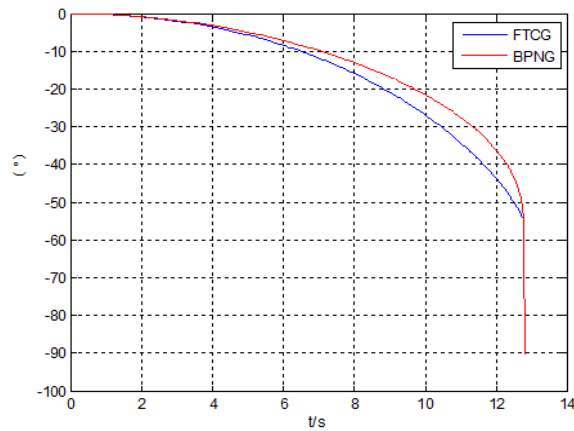

Fig.4 Angle of the line of sight

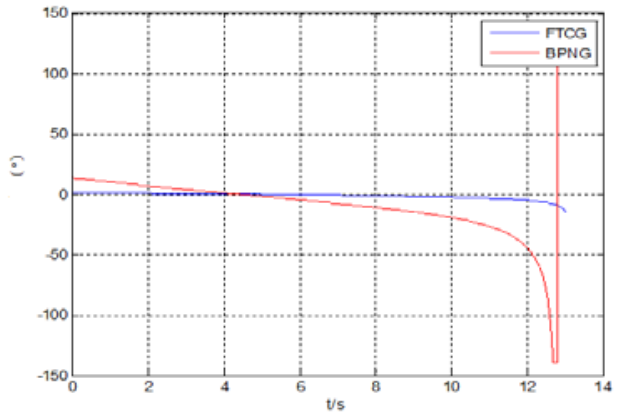

Fig. 5 Missile overload schematic

Can be seen from figure 2, missile guidance in the last stage, compared FTCG BPNG has a straighter flight trajectory, the size of the missile precision and speed is very beneficial, to attack the ground fixed target or the passive strapdown homing missile ground fortifications, flat trajectory for precise regulation of terminal guidance. By figure 3 shows two guidance law of convergence than expected Angle, elevation Angle are basic to realize the function of the guidance law is designed. From figure 4, you can see that the guidance law is designed in this paper FTCG in convergence condition when a missile hit the target, and at the time of the terminal guidance BPNG divergence Angle of the line of sight, lead to the stability of the missile, greatly influenced the performance of the guidance, the serious influence missile accuracy. Can be seen from figure 5, FTCG overload are much smaller than actual application overload, and the actual overload BPNG overload is greater than the set of available. The designed guidance law from the perspective of a missile motor overload is easy to implement, ensure the missile in the process of flying to the target to maintain the stability of the strong. Based on analysis, this paper design the guidance law of performance due to the traditional offset proportional guidance law, has stronger robustness and better.

\section{Summary}

Fall for missile terminal guidance Angle constraint problem, this paper selected the linear sliding mode surface, designed a kind of finite time convergence with fall Angle constraint sliding-mode guidance law, analyze the finite time and proves that the and to weaken the chattering phenomenon analysis, through simulation comparison, in this paper, the guidance law designed simple form, has strong robustness and good guidance performance, has wide research space and prospect.

\section{References}

[1] Kim M, Grider k. Terminal guidance for impact attitude Angle constrained flight trajectories [J]. IEEE the Transactions on Aerospace and Electronic Systems, 1973, 9 (6) : 852-859.

[2], scene, tang jiao, etc. A biased proportional guidance law based on fall Angle constraint [J]. Journal of Beijing institute of technology, 2014, 34 (3) : 277-282.

[3] zhang xu, hu-min lei, Ceng Hua XiaoZengBo, Ye Jikun, etc. With fall Angle constraint adaptive proportional guidance law [J]. J solid rocket technology, 2011, 34 (6) : 687-692.

[4] Xiong Shaofeng, wei-hong wang, liu xd, sen, wang wu. Considering the dynamic characteristics of missile autopilot with attack Angle constraint guidance law [J]. Control and decision, 2015, 30 (4) : 585-592. 
[5] Zhang Yunxi, Sun Mingwei, Zengqiang variable structure guidance law for finite time convergence [J] Control Theory and Applications, 2012, 29 (11): 1413-1418. 